\title{
Optimal Performance State Estimation of Compute Systems
}

\author{
Michael Manzke and Brian Coghlan \\ Department of Computer Science, University of Dublin, Trinity College, Ireland \\ michael.manzke@cs.tcd.ie and brian.coghlan@cs.tcd.ie
}

\begin{abstract}
This paper presents a novel approach to estimating and predicting the system-wide utilisation of computational resources in real-time. An algorithm that implements a discrete minimum mean-square error filter is applied to fuse concurrent and sequential observations of system event counts into a state vector. Contemporary computer components and subsystems make these event counts available through hardware performance counter registers. The registers may be accessed by the system's software quasiconcurrently but the number of registers in individual components is usually smaller than the number of events that can be monitored. Our approach overcomes this problem by modeling individual hardware performance counter readings as vector random processes and recursively processes them one at a time into a common state vector, thereby making larger performance counter sets observable than would otherwise be possible.
\end{abstract}

\section{Introduction}

Todays high performance computers, whether singleprocessor or multi-processor systems, are made-up of concurrently operating sub-systems. The run-time knowledge of the system-wide utilisation state of these subsystems including CPUs and their interconnects can assist efficient scheduling of tasks onto these resources. This paper is not concerned with the scheduling aspect but introduces an algorithm that generates a global view of the system's utilisation. These observations are derived from hardware performance counter readings that count the number of occurrences of a selected event.

The performance counter register reading $z_{k}$ in Eq.(1) represents the mean of the counted events $e(t)$ over the sample interval $\Delta t$. The choice of duration of the sample interval $\Delta t$ is a trade-off between counter accuracy and computational overhead. Reducing $\Delta t$ will increase the accuracy until the computational overhead (caused by instruc- tions that read the counter registers) contributes a significant amount of counter events to the counter reading.

$$
z_{k}=\frac{1}{\Delta t} \sum_{t=k \Delta t}^{(k \Delta t)+\Delta t} e(t)
$$

System events such as the Number of Instruction Fetch Misses that are counted over a time period provide a measurement of the degree of availability or utilisation of particular system resources. These observations are easily obtained through registers that implement performance counters. In general counter registers can be instructed to count a particular event by means of a selection register. This selection is required because in most cases the number of registers does not match the number of events that may be counted [2].

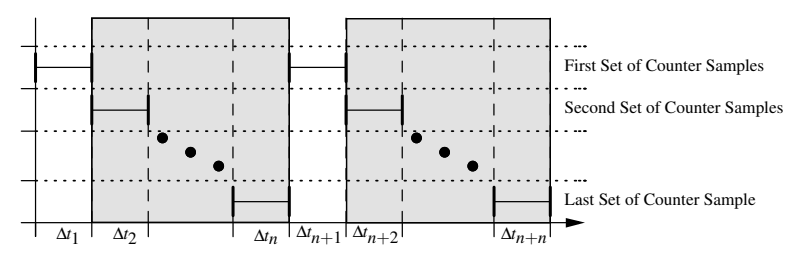

Figure 1 . Multiplexed sets of performance
counter readings

If the number of distinct event counts exceeds the number of available registers then this approach fails. One remedy is if the system uses a single register to count different events in a nested sequence. Fig. 1 depicts this multiplexing technique. A set in Fig. 1 refers to the number of performance counter registers in a given sub-system. The shaded areas in this figure highlight the intervals $\left[\Delta t_{2}, \Delta t_{n}\right]$ and $\left[\Delta t_{n+2}, \Delta t_{n+n}\right]$ with $n$ as the total number of event sets required to observe the current state of resource utilisation. No events are counted for the first counter set, and consequently the accuracy decreases for these multiplexed readings [1]. 
This papers proposes to model performance counter readings as random processes and to apply a Kalman filter [3] to fuse performance counter readings into a statevector that holds an optimal estimate of the system-wide event counts. This discrete minimum mean-square error filter was originally formulated by R. Kalman in 1960 and can process multiple time-variable inputs through the use of state space methods. The application of stochastic system models for the performance counter readings is not only beneficial for the optimal estimation of a noise-corrupted and incomplete scalar reading but also to multiple readings.

\section{The Algorithm}

Our approach can provide a system-wide optimal estimate of the performance counter readings that may be merged into a filter's state vector as subsets of the total set of counter observations. The system software that implements the acquisition and processing of performance counter register readings introduces additional system dynamics and distortions. This software includes firstly a driver that provides access to the registers, secondly a lowlevel user-space API that implements the control of the registers and finally the filter algorithm itself [4]. The computer's architecture and operating system design can result in non-determinism in the execution time of the above mentioned software; this leads to a noise corruption of the reading that is otherwise only corrupted by quantisation noise.

The filter's objective is to provide an optimal to suboptimal estimation and prediction of $n$ performance counter readings. This estimate or prediction is based on imperfect and incomplete counter data; imperfect if corrupted by quantisation noise and variations in the counter acquisition process, and incomplete if counter readings are multiplexed. A Kalman filter uses stochastic models to accommodate these properties. Stochastic models can overcome problems arising from imperfect system models and noise that cannot be modeled deterministically.

The filter incorporates the system dynamics into the model. In its fundamental form the model is assumed to be linear and driven by white Gaussian noise and the performance counter readings that are taken from this process are also assumed to be corrupted by white Gaussian noise. These measurements are presumed to have a linear relationship with the system model. Note that an Extended Kalman Filter $(E K F)$ can be used if either the system model is nonlinear or the measurements are not linearly related with the system model. However, a linear Kalman Filter proves adequate for the observation of performance counter readings.

The filter is a recursive algorithm that processes measurements (in our case performance counter readings) of any quality in order to derive an optimal estimate from these data. The algorithm must be fed with information about the process and measurement dynamics. Furthermore, statistics about the measurement noise and the process model's uncertainty must be supplied to the filter algorithm along with an initialisation. Fig. 2 demonstrates the recursive nature of the algorithm. Fig. 2 shows also one possible implementation, but there are algebraically equivalent forms that have computational advantages and disadvantages.

The filter provides an optimal estimate by combining the measurements and the knowledge of the system to minimise the error between the model and the measurements statistically. To this end the algorithm propagates the conditional probability density of the system's state. The probability density is conditioned by the discrete time performance counter samples and represents the uncertainty of the current state of the system.

The Kalman Filter algorithm is presented in Fig. 2 and the discrete Kalman Filter matrix block diagram in Fig. 3 will help us to discuss the operations of the filter.

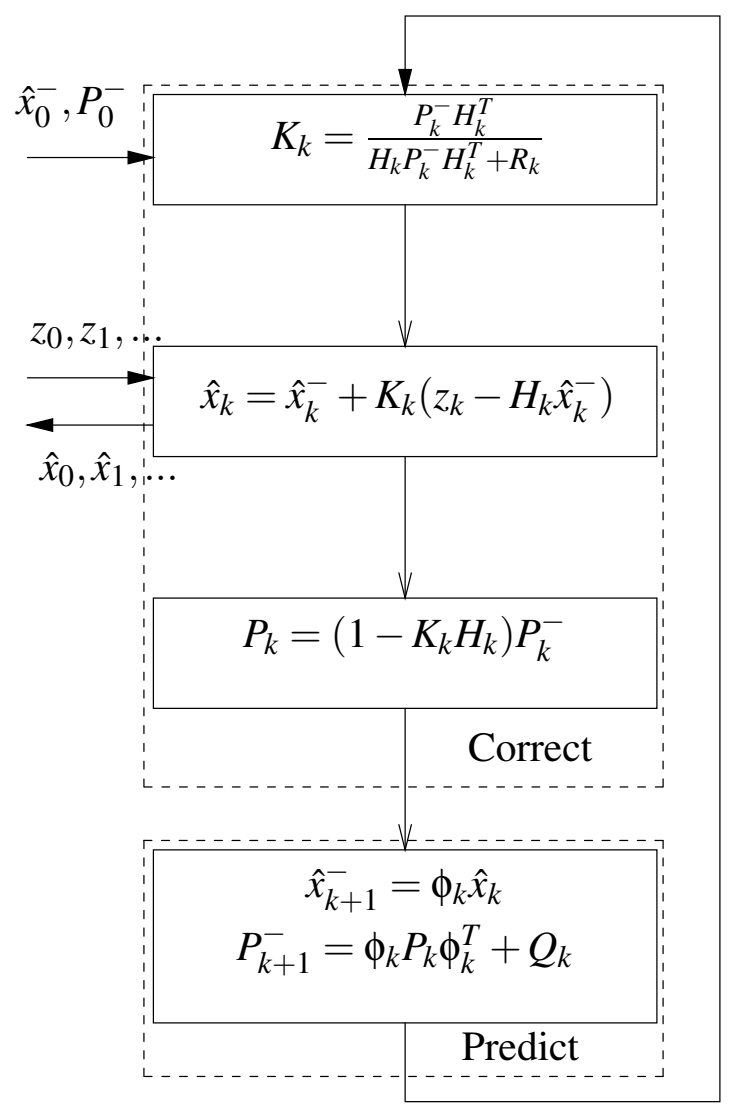

Figure 2. Discrete Kalman Filter Algorithm 


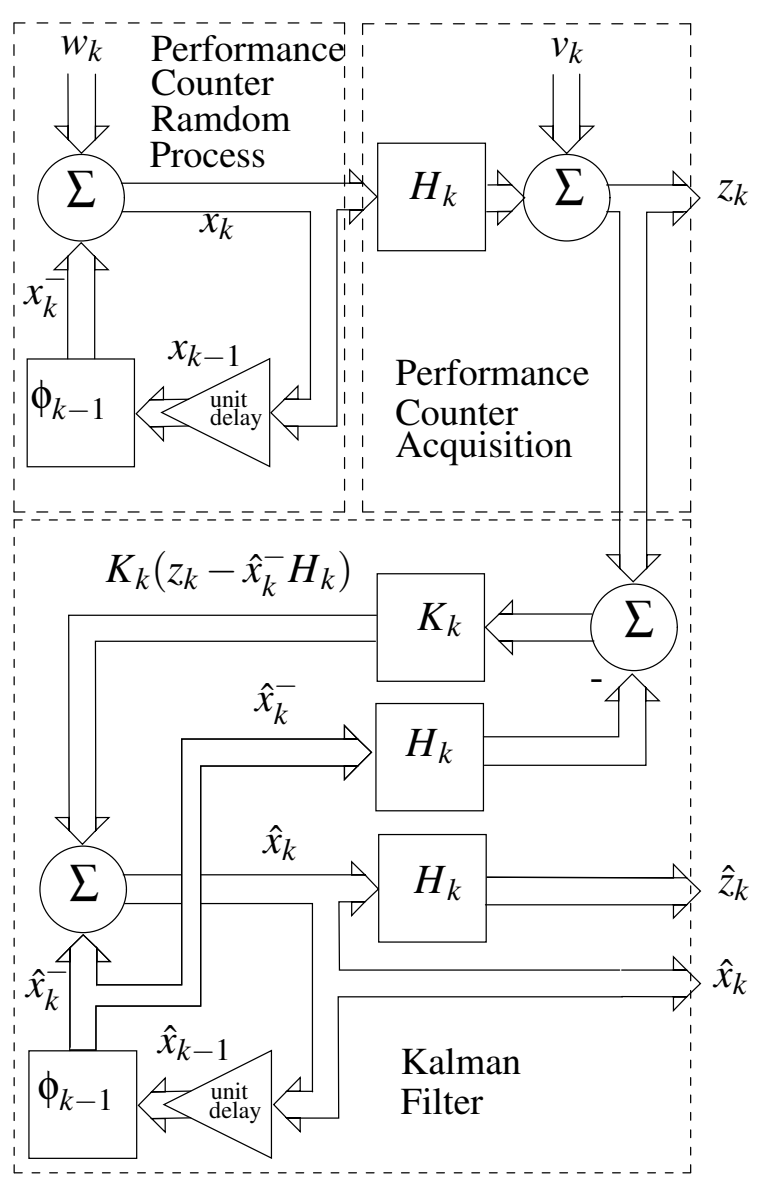

\section{Figure 3. Discrete Kalman Filter Matrix Block Diagram}

\subsection{Performance Counter Process Models}

The Kalman Filter implementation requires the performance counter readings to be modeled as random processes. Both a Gauss-Markov Process and antegrated Gauss-Markov Process were investigated as a suitable random process model. Limited investigation indicated that an integrated Gauss-Markov process represents a performance counter random process more accurately.

A stationary Gaussian process $X(t)$ that has an exponential autocorrelation function is classified as a GaussMarkov process. Eq.(2) defines the autocorrelation function for a Gauss-Markov process.

$$
R_{X}(\tau)=\sigma^{2} e^{-\beta|\tau|}
$$

This autocorrelation function Eq.(2) provides the full statistical description of the random process. The two parameters, the time constant $\frac{1}{\beta}$ and the variance $\sigma^{2}$ can be

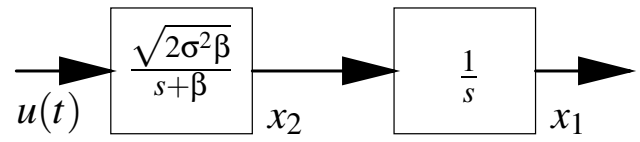

Figure 4. Integrated Gauss-Markov Block Diagram

calculated from an experimentally determined autocorrelation function. Fig. 4 shows the transfer function for an integrated Gauss-Markov process. With $u(t)$ as unity white noise, $x_{2}$ as Gauss-Markov process and $x_{1}$ as integrated Gauss-Markov process.

We assume that every hardware counter reading is taken from an independent random counter process $i$ with a specific autocorrelation function. This allows us to define the discrete state transition matrix $\phi$ and the discrete covariance matrix $Q$ for $n$ counters.

With the filter algorithm and the process model in place we can now continue to calculate the time constants $1 / \beta_{i}$ and the variances $\sigma_{i}^{2}$ for individual performance counters. To this end we use Mikael Pettersson's [4] Linux x86 Performance-Monitoring Counters Driver to sample performance counter readings for a subsequent offline analysis. The sampled performance counter readings are processed with Eq.(3) to calculate the estimated autocorrelation function $\hat{R}_{X}(n \Delta t)$ with $N$ as the total number of samples. A curve fitting computation provides us with the time constants $1 / \beta_{i}$ and the variances $\sigma_{i}^{2}$. Fig. 5 shows the mean of 10 estimated autocorrelation functions as $R_{x}$ and the fitted curve $\left(\sigma^{2} e^{(-\beta \Delta t)}\right)+$ mean $^{2}$.

$$
\hat{R}_{X}(n \Delta t)=\frac{1}{N-n+1} \cdot \sum_{k=0}^{N-n} X(t) X(k+n)
$$

\subsection{One Performance Counter Set at a Time}

In order to process sets of performance counters one-ata-time we must make modifications to the recursive filter algorithm presented in Fig. 2. The three equations for the Kalman gain $K_{k}$, the posteriori state estimate $\hat{x}_{k}$ and the error covariance $P_{k}$ are all part of the filter's correction process. In the modified algorithm the equation performs the correction only on submatrices that are associated with the available performance counter set for the current iteration of the loop. This process allows for the merging of available counter readings into the state vector $\hat{x}_{k}$. The remaining two equations of the filter algorithm, the priori state vector $\hat{x}_{k+1}^{-}$and the priori covariance matrix $P_{k+1}^{-}$implement the prediction aspect of the filter. This part of the al- 


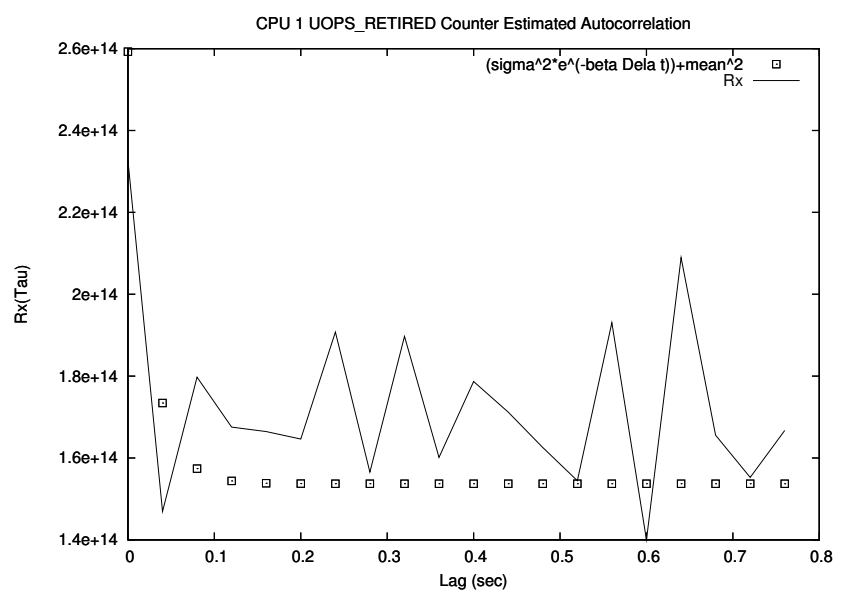

Figure 5. Estimated autocorrelation functions and fitted exponential curve for the event: Number of microops retired on CPU1 of a two-way PIII SMP system.

gorithm remains unchanged. Consequently the entire state vector $\hat{x}$ for all the performances counters is propagated according to the state transition matrix $\phi$. This is an important detail because the state vector $\hat{x}$ and (through the measurement matrix $H$ ) the estimated measurement $\hat{z}$ changes over the time intervals without data according to the system dynamics defined in the state transition matrix $\phi$. Furthermore the priori covariance matrix $P_{k+1}^{-}$propagates the uncertainty for counter processes during every iteration of the algorithm. Therefore the measure of uncertainty for unavailable counters will increase with every iteration until new data are available. Also an optimal blending operation can be performed once data become available. This is in contrast to a sample-and-hold operation.

\section{Experimental Evaluation}

Fig. 6 demonstrates the performance of the one-at-atime filter solution. The algorithm fuses three sets of performance counters into the state vector. The figure shows only one counter and the associated state variables. In Fig. 6 the state variable $x_{1}$ estimates the events/sec from the noisy counter readings despite the fact that only every third sample interval provides data for the filter's blending operation. It is obvious that the filter does not trust the counter readings entirely hence the difference between state variable $x_{1}$ and the counter readings.

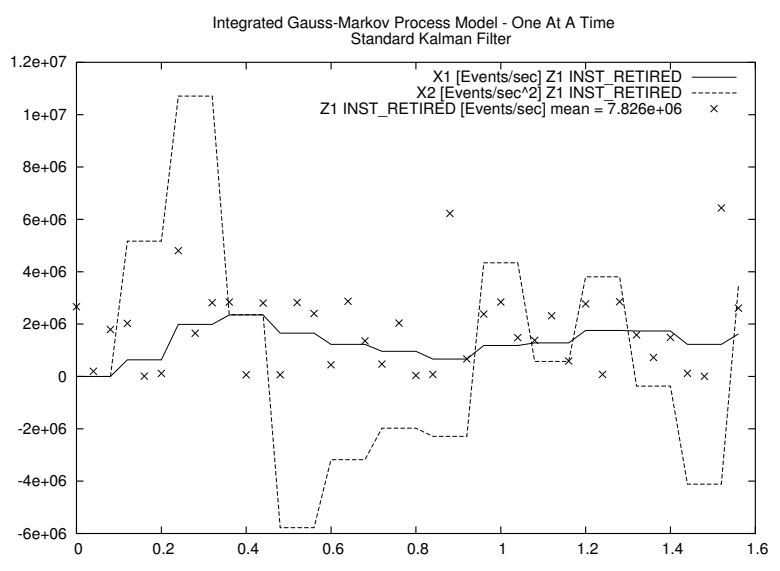

Figure 6. One-at-time Filter with integrated Gauss-Markov process model shows two state variables and a performance counter reading.

\section{Conclusions}

In this paper we demonstrate that a discrete minimum mean-square error filter may be successfully applied to fuse concurrent and sequential observations of system event counts into a state vector. This technique allows the observation of an increased variety of events without sacrificing accuracy. The optimal estimate of large sets of performance counter processes provides us with the means to observe complex systems by combining the elements of the state vector to infer system metrics that are unobservable with a restricted number of counter readings.

\section{References}

[1] J. Dongarra, K. London, S. Moore, P. Mucci, and D. Terpstra. Using papi for hardware performance monitoring on linux systems. In Conference Proceedings of Linux Clusters: The HPC Revolution, Urbana, Illinois, jun 2001.

[2] Intel. IA-32 Intel Architecture Software Developer's Manual, 2001.

[3] E. Kalman, Rudolph. A new approach to linear filtering and prediction problems. Transactions of the ASME-Journal of Basic Engineering, 82(Series D):35-45, 1960.

[4] M. Pettersson. Linux x86 performance-monitoring counters driver. http:www.csd.uu.se mikpelinuxperfctr, jan 2002. 